\title{
Dor crônica e nível de atividade física em usuários das unidades básicas de saúde
}

\section{Chronic pain and level of physical activity in users from basic health units}

\section{AUTORES \\ Daniele Fernandes da Silva de Souza ${ }^{1,2,3}$ (D) \\ Vítor Häfele ${ }^{1,2,3}$ (D) \\ Fernando Vinholes Siqueira ${ }^{1,2,3}$ (D) \\ 1 Escola Superior de Educação Física da Universidade Federal de Pelotas, Pelotas, Rio Grande do Sul, Brasil. \\ 2 Programa de Pós-Graduação em Educação Física da Universidade Federal de Pelotas, Pelotas, Rio Grande do Sul, Brasil. \\ 3 Grupo de Estudos em Epidemiologia da Atividade Física da Universidade Federal de Pelotas, Pelotas, Rio Grande do Sul, Brasil}

\section{CONTATO}

Daniele Fernandes da Silva de Souza dan2nha@gmail.com

Rua Luis de Camões, 625, Pelotas, Rio Grande do Sul, Brasil.

CEP: 96055-630.

DOI

$10.12820 /$ rbafs. $24 \mathrm{e} 0085$

\begin{abstract}
RESUMO
O objetivo do estudo foi descrever a prevalência de dor crônica dos usuários das unidades básicas de saúde da zona urbana da cidade de Pelotas, Rio Grande do Sul, Brasil, e verificar o nível de atividade física e os fatores associados à dor crônica. Foi realizado um estudo transversal com amostra de 540 adultos com 18 anos ou mais, usuários de 36 unidades básicas de saúde. Considerou-se dor crônica aos que relataram o tempo referido de algum tipo dor de três meses ou mais. Foram estimadas razões de prevalência ajustada por meio da regressão de Poisson, com variância robusta. A prevalência de dor crônica entre os usuários foi de 41,5\% (IC95\%: 37,4 - 45,7). Na análise ajustada, as mulheres tiveram 50\% maior probabilidade ao desfecho em relação aos homens; indivíduos de 40-59 anos e 60-90 anos, tiveram maior probabilidade de 44\% e 61\%, respectivamente, do que os de 18-39 anos; aqueles que perceberam sua saúde negativamente, tiveram 5,4 vezes mais probabilidade ao desfecho; utilizar três ou mais medicamentos aumentou $43 \%$ a probabilidade ao desfecho e; sujeitos que praticavam atividade física de lazer tiveram menor probabilidade de apresentar dor crônica. Conclui-se que, a prevalência de dor crônica em usuários de unidades básicas de saúde foi elevada (41,5\%), sendo que a atividade física de lazer foi fator de proteção para o surgimento de dor crônica. Contudo, para o tratamento da dor crônica nas unidades básicas de saúde, a prática de atividade física torna-se de fundamental importância.
\end{abstract}

Palavras-chave: Dor crônica; Atividade motora; Unidades básicas de saúde.

ABSTRACT

The aim of the study was to describe the prevalence of chronic pain among users of basic health units in the urban area of the city of Pelotas, Brazil, and to verify the level of physical activity and the associated factors with chronic pain. A cross-sectional study was performed with 540 adults 18 years and older, users of 36 basic health units. Considered chronic pain was referred time of some kind of pain of three months or more. Adjusted prevalence ratios were estimated using Poisson regression, with robust variance. The prevalence of chronic pain among users was 41.5\% (CI95\% 37.4 - 45.7). In the adjusted analysis women had 50\% more likely of outcome compared to men; individuals aged 40-59 years and 60-90 years bad more likely of 44\% and 61\%, respectively, than those aged 18-39 years; those who perceived their health negatively were 5.4 times more likely to the outcome; using three or more drugs increased the likely of outcome by $43 \%$ and; leisure physical activity was a protective factor for the onset of chronic pain. It is concluded that the prevalence of chronic pain in user of basic health units was elevated (41,5\%), being that the leisure physical activity was protection factor for the onset of chronic pain. However, for the treatment of chronic pain in basic health units, the practice of physical activity becomes of fundamental importance.

Keywords: Chronic pain; Motor activity; Basic health unit.

\section{Introdução}

As Unidades Básicas de Saúde (UBS) desempenham um papel fundamental na oferta à população no acesso em saúde de qualidade através da atenção básica. A atenção básica, no Brasil, é desenvolvida de forma descentralizada e deve ser a principal porta de entrada e centro de comunicação do usuário com toda a rede de atenção à saúde do Sistema Único de Saúde (SUS) ${ }^{1}$.

Dentre os motivos de procura de atendimento do SUS a dor crônica é uma frequente razão para consulta ${ }^{2}$. Revisão sistemática realizado por Jackson et al. ${ }^{3}$, identifi- cou que, a prevalência de dor crônica variou de 5\% a 42\% em uma população geral de adultos, de $5 \%$ a $62 \% \mathrm{em}$ uma população geral de idosos e de $10 \%$ a $79 \%$ em trabalhadores. $\mathrm{Na}$ população geral do Brasil, a prevalência de dor crônica variou de $35,5 \%$ a $42 \%{ }^{3}$. No entanto, em países em desenvolvimento como o Brasil, são necessários estudos mais detalhados sobre a sua real ocorrência ${ }^{4}$.

Pelo fato da dor crônica não ser um mero prolongamento da dor aguda, sua natureza é distinta, havendo a adaptação dos sistemas neuronais e disfunção prolongada do sistema nervoso periférico, sistema nervoso central 
ou de ambos ${ }^{5}$, podendo durar por meses e até por anos ${ }^{6}$.

Pesquisas a nível mundial têm revelado que a dor crônica está associada a alguns fatores, tais como ser do sexo feminino e a alterações de saúde mental, assim como muitos dos que referem dor crônica, têm sido diagnosticados com depressão por causa da dor ${ }^{6,7} . \mathrm{Na}$ maioria das pessoas, a dor crônica afeta severamente o sono, a capacidade de se exercitar, andar, realizar tarefas domésticas, participar de atividades sociais, manter um estilo de vida independente, e a capacidade de ter relações sexuais e a manutenção das relações familiares ${ }^{7}$.

Algumas medidas podem ser utilizadas como forma de prevenção e tratamento da dor crônica, dentre elas a prática de atividade física, a qual proporciona melhores condições através do reconhecimento científico e da ajuda terapêutica que ela promove ${ }^{8}$. A atividade física favorece a diminuição da gordura corporal e a redução dos marcadores sistêmicos de inflamação, o que também contribui para a diminuição da dor crônica9

Com o objetivo de promover o aumento da prática de atividade física na população, consta nos planos do Ministério da Saúde, a atuação dos profissionais de educação física no cotidiano da rede básica de saúde ${ }^{10}$, tais como as suas amplas possibilidades de intervenção no Núcleo de Apoio à Saúde da Família (NASF) através de atividades como exercícios de alongamentos, fortalecimento muscular, caminhada, coordenação motora e atividades lúdicas ${ }^{11}$, visando o aumento da saúde, qualidade de vida e, consequentemente, diminuição do impacto causado pelas dores crônicas nos usuários ${ }^{12}$.

No entanto, os estudos abordando a temática dor crônica e fatores associados em usuários de UBS são escassos, principalmente relacionando com a prática de atividade física, sendo os estudos existentes conduzidos em poucas $\mathrm{UBS}^{12}$. Além disso, no caso deste estudo, a dor crônica é abordada de forma ampla, não se detendo a uma localização específica da dor ou população específica, mas a uma população que sente dor e procura uma solução através da UBS mais próxima na sua comunidade. Portanto, o estudo teve como objetivo descrever a prevalência de dor crônica e sua associação com o nível de atividade física, variáveis socioeconômicas, demográficas, comportamentais, nutricional e de saúde dos usuários de UBS da zona urbana da cidade de Pelotas, Rio Grande do Sul.

\section{Métodos}

Foi realizado um estudo transversal de base populacional comunitária com usuários de UBS da zona urbana da cidade de Pelotas, Rio Grande do Sul, aprovado pelo Comitê de Ética em Pesquisa da Escola Superior de Educação Física da Universidade Federal de Pelotas (ESEF-UFPel), (Protocolo 2.496.718). Após a autorização da Secretaria Municipal de Saúde, foi estabelecido contato com os responsáveis das UBS para o agendamento da data da coleta de dados. Foi solicitado a cada entrevistado, previamente a entrevista, a assinatura do termo de consentimento livre e esclarecido, informado ao mesmo o direito a não participação e a garantia de confidencialidade das informações fornecidas.

O cálculo do tamanho da amostra foi realizado considerando uma a prevalência de dor crônica em 45\%; nível de confiança de 95\%, totalizando uma amostra de 412 adultos. Com um acréscimo de $10 \%$ para perdas e recusas e de $15 \%$ para análise multivariável, resultou em 524 adultos. De forma a atingir a cota de 15 adultos em cada UBS, chegou-se a uma amostra final de 540 adultos.

Foram entrevistados adultos de 18 anos ou mais, de ambos os sexos e consideradas para a composição da amostra todas as 38 UBS da zona urbana da cidade. Optou-se por excluir da amostra duas UBS que atendem somente populações exclusivas.

Os usuários foram abordados individualmente para as entrevistas, enquanto aguardavam atendimento na sala de espera, começando sempre pelo primeiro à direita da sala, partindo da porta de entrada, continuando pelo seguinte à sua esquerda e assim sucessivamente, até que fosse atingida a cota predeterminada de 15 usuários.

As entrevistas foram realizadas por cinco estudantes (graduação e pós-graduação) da UFPel, fizeram parte da coleta de dados, a qual ocorreu entre os meses de março e junho de 2018. Todos os entrevistadores possuíam experiência prévia em coleta de dados em estudos populacionais e receberam capacitação sobre assuntos pertinentes ao estudo realizados pela pesquisadora responsável, em um período de quatro horas.

As variáveis independentes incluídas na análise foram: sexo (masculino e feminino), faixa etária (18-39 anos: adultos jovens; 40-59 anos: meia idade e; 60-90 anos: idosos), cor da pele (branca e não branca, observado pelo entrevistador), situação conjugal (com e sem companheiro), renda mensal individual em salários mínimos (menos de 1 salário, de 1 até 2 salários e mais de 2 salários), situação profissional (aposentado, não trabalha e trabalha), tabagismo (nunca fumou, ex-fumante e fumante), índice de massa corporal - IMC, determinado por meio do peso e altura autoreferidos, autopercepção de saúde (excelente/muito boa, boa, regular e 
ruim), diagnóstico médico de: hipertensão arterial (não e sim), diabetes mellitus (não e sim) e depressão (não e sim); número de medicamentos referidos para uso contínuo (nenhum, entre 1 ou 2 medicamentos e 3 ou mais), atividade física de lazer (fisicamente inativo: $\leq 10$ minutos/semana, insuficientemente ativo: de 10 a 149 minutos/semana e fisicamente ativo: $\geq 150$ minutos por semana) e atividade física de trabalho (fisicamente inativo: $\leq 10$ minutos, insuficientemente ativo: de 10 a 149 minutos/semana e ativo: $\geq 150$ minutos/emana) ${ }^{13}$. As variáveis atividade física de lazer e trabalho foram coletadas por meio do questionário internacional de atividade física (IPAQ) na versão longa, considerando uma semana típica.

O desfecho, dor crônica, foi operacionalizado através da pergunta: “O(A) Sr.(a), esta semana, está sentindo alguma dor? Se a resposta fosse sim, foi perguntado: Há quanto tempo o(a) Sr.(a) sente esta dor? Com quatro opções de resposta: Menos de 1 mês, de 1 mês a menos de 3 meses, de 3 meses a menos de 6 meses ou há mais de 6 meses?" Foi considerado, para este estudo, como ponto de corte para dor crônica, o tempo de três meses ou mais referido de dor, por ser o critério utilizado pela Associação Internacional para Estudos da Dor (IASP), tanto na população geral, quanto na atenção primária ${ }^{14}$.

Para a avaliação da intensidade da dor foi utilizada a escala de faces de Wong e Baker ${ }^{15}$, a qual exibe a imagem de seis faces: "0" sem dor (expressão feliz) " 1 " dor leve (expressão não muito feliz), "2" dor moderada (expressão não sorridente), “3” dor forte (expressão triste), “4" dor insuportável (expressão bem triste) e " 5 " dor máxima (expressa um rosto extremamente triste, choroso). A intensidade da dor foi avaliada no período da cronicidade (mais de 3 meses) e no momento da entrevista.

Para avaliar a dor na sua multidimensionalidade, foi considerada a sua interferência na atividade geral, na qualidade do sono, no humor, no trabalho, na habilidade para caminhar, no relacionamento com outras pessoas e para apreciar a vida, onde: "0" não interfere, " 1 " interfere um pouco, " 2 " interfere moderadamente e " 3 " interfere totalmente, segundo o questionário breve de dor de Wisconsin ${ }^{6}$. As regiões álgicas mais afetadas pela dor, foram localizadas através da imagem de um corpo humano de frente e outra de costas (poderiam ser localizadas até três regiões álgicas por entrevistado). $\mathrm{O}$ instrumento também avaliou quanto a realização de tratamentos para alívio da dor (não e sim), os tratamentos utilizados, faltas ao trabalho devido a dor (não e sim), uso de medicamentos para dor (não e sim), a porcentagem que obteve de alívio no tratamento ou uso de medicação para a dor $(0 \%, 10 \%, 20 \%, 30 \%, 40 \%$, $50 \%, 60 \%, 70 \%, 80 \%, 90 \%$ e $100 \%)^{6}$.

Questões relacionadas ao tempo (meteorologia), também foram consideradas: foi perguntado a temperatura a qual percebe mais dor (mais elevada, mais baixa ou não tem relação) e o clima o qual percebe mais dor (seco, úmido ou não tem relação) ${ }^{16}$.

$\mathrm{O}$ banco de dados foi elaborado no programa Epidata 3.1 e para a análise dos dados utilizou-se o programa Stata 13.1. Na análise descritiva foram incluídas as variáveis socioeconômicas, demográficas, comportamentais, nutricional e de saúde. O teste do Qui-quadrado foi utilizado para comparar o desfecho dor crônica com cada variável independente. Foram estimadas razões de prevalência por meio da Poisson com variância robusta, com intervalos de confiança de $95 \%$. O teste de Wald foi utilizado para verificar heterogeneidade e tendência linear. $\mathrm{Na}$ análise multivariável, considerou-se um modelo teórico de determinação com blocos hierarquizados: $1^{\circ}$ nível - sexo, idade, cor da pele, situação conjugal e renda mensal (salários mínimos); $2^{\circ}$ nível - tabagismo; $3^{\circ}$ nível - situação profissional; $4^{\circ}$ nível - índice de massa corporal e auto-percepção de saúde; $5^{\circ}$ nível - diagnóstico médico referido de hipertensão arterial, diabetes e depressão; $6^{\circ}$ nível - número de medicamentos referidos para uso contínuo; $7^{\circ}$ nível - atividade física de lazer e atividade física de trabalho. As atividades físicas de lazer e trabalho, foram adicionadas separadamente ao modelo, devido ao efeito particular que cada uma exerce ao controlar as demais variáveis. Portanto, montou-se apenas um modelo, onde ao final do mesmo foi acrescentada a atividade física de lazer e após a anotação dos seus valores, a mesma foi retirada e acrescentou-se a atividade física de trabalho. Todas as variáveis com valor de $\mathrm{p} \leq 0,20$ foram mantidas no modelo de regressão com o propósito de controlar para possíveis fatores de confusão.

\section{Resultados}

Foram entrevistados 540 usuários de 36 UBS da zona urbana da cidade de Pelotas, Rio Grande do Sul. Houve 53 recusas e não foram registradas perdas, tendo em vista que os questionários não concluídos foram substituídos. A maioria da amostra foi composta por mulheres (72,8\%; IC95\%: 68,8 - 76,3), pessoas de cor de pele branca (72,4\%; IC95\%: 68,4 - 76,0) e que viviam com companheiro(a) (59,4\%; IC95\%: 55,1 - 63,4). A média de idade foi de 48,0 $\pm 17,2$ anos (variando de 18 
a 90 anos). Em relação a situação profissional, 42,8\% (IC95\%: 38,6 - 47,0) não trabalhavam, dos usuários que trabalhavam, 55,6\% (IC95\%: 48,9 - 62,0) eram ativos fisicamente no trabalho. Quanto à renda mensal em salários mínimos, 38,2\% (IC95\%: 34,0 - 42,2) recebia menos de um salário e 50,8\% (IC95\%: 46,6 $55,0)$ recebia de 1 até 2 salários. Em relação ao nível de atividade física no lazer 70,8\% (IC95\% 66,7 - 74,4) eram inativos. Quanto ao tabagismo, 20,2\% dos entrevistados eram fumantes (IC95\%: 17,0 - 23,7). Aproximadamente $50 \%$ dos usuários perceberam a sua saúde como excelente, muito boa ou boa. Quanto ao recebimento de diagnóstico médico, 42,2\% (IC95\%: 38,1 - 46,4) relataram hipertensão arterial, 13,9\% (IC95\%: 11,2 - 17,0) diabetes mellitus e 28,0\% (IC95\%: 24,3 - 31,9) depressão. Três em cada dez adultos (30\%; IC95\%: 26,1 - 34,0) foram classificados com obesidade. Cerca de 63\% (IC95\%: 59,4 - 67,5) dos usuários relataram utilizar um medicamento ou mais de uso contínuo (Tabela 1).

A prevalência de dor crônica foi de 41,5\% (IC95\%: 37,4 - 45,7). Dentre os usuários com dor crônica, $62,9 \%$ relataram a intensidade da dor como forte, insuportável ou máxima no período da cronicidade e no momento da entrevista $65,7 \%$ relataram estar com intensidade leve ou sem dor. A dor interferiu totalmente na atividade geral em 34,4\% (IC95\%: 28,4 - 40,8) dos relatos, também interferiu totalmente na qualidade do sono em 32,6\% (IC95\%: 26,7 - 39,0), no trabalho 35,7\% (IC95\%: 26,7 - 45,8) e na habilidade para caminhar em 33,9\% (IC95\%: 27,9 - 40,4) dos entrevistados. Em relação à tratamentos para alívio da dor, 74,1\% (IC 95\%: 67,9 - 79,4) dos usuários relataram utilizar algum tipo de tratamento, sendo que, $82,5 \%$ utilizavam tratamento medicamentoso. As faltas ao trabalho por causa da dor foram relatadas em 69,0\% (IC95\%: 59,1 - 77,4) dos entrevistados que estavam trabalhando. Os medicamentos para dor foram utilizados em 84,8\% (IC95\%: 79,4 $88,9)$ dos usuários e a maioria relatou que a medicação ou tratamento para dor aliviou em no mínimo $50 \%$ a sensação de dor. Cerca de 44,0\% e 49,0\% percebiam que temperaturas mais baixas e o clima úmido, respectivamente, tinham relação com a dor crônica (Tabela 2).

As regiões apontadas pelos usuários como as mais afetadas pela dor foram: lombar $(28,6 \%)$, joelhos $(21,0 \%)$, cabeça $(14,3 \%)$, cervical $(13,4 \%)$, ombros $(8,9 \%)$ e torácica $(8,9 \%)$.

$\mathrm{Na}$ análise bruta, ser do sexo feminino, possuir idade avançada, auto percepção de saúde negativa, diag-
Tabela 1 - Descrição das variáveis socioeconômicas, demográficas, comportamentais, nutricionais e de saúde dos usuários das unidades básicas de saúde da zona urbana da cidade de Pelotas, Rio Grande do Sul, $2018(\mathrm{n}=540)$.

\begin{tabular}{|c|c|c|c|}
\hline Variáveis & $\mathrm{n}$ & $\%$ & IC 95\% \\
\hline \multicolumn{4}{|l|}{ Sexo } \\
\hline Masculino & 147 & 27,2 & $23,6-31,1$ \\
\hline Feminino & 393 & 72,8 & $68,8-76,3$ \\
\hline \multicolumn{4}{|l|}{ Faixa etária (anos) } \\
\hline $18-39$ & 179 & 33,2 & $29,3-37,3$ \\
\hline $40-59$ & 213 & 39,5 & $35,4-43,7$ \\
\hline $60-90$ & 147 & 27,3 & $23,6-31,2$ \\
\hline \multicolumn{4}{|l|}{ Cor da pele } \\
\hline Branca & 391 & 72,4 & $68,4-76,0$ \\
\hline Não-branca & 149 & 27,6 & $23,9-31,5$ \\
\hline \multicolumn{4}{|l|}{ Situação conjugal } \\
\hline Sem companheiro(a) & 219 & 40,6 & $36,5-44,8$ \\
\hline Com companheiro(a) & 320 & 59,4 & $55,1-63,4$ \\
\hline \multicolumn{4}{|l|}{ Renda mensal (salários mínimos) } \\
\hline Menos de 1 & 205 & 38,2 & $34,0-42,2$ \\
\hline De 1 até 2 & 274 & 50,8 & $46,6-55,0$ \\
\hline 2,01 ou mais & 60 & 11,0 & $8,7-14,0$ \\
\hline \multicolumn{4}{|l|}{ Situação profissional } \\
\hline Aposentado & 110 & 20,4 & $17,1-23,9$ \\
\hline Não trabalha & 231 & 42,8 & $38,6-47,0$ \\
\hline Trabalha & 199 & 36,8 & $32,8-41,0$ \\
\hline \multicolumn{4}{|l|}{ Atividade física (lazer) } \\
\hline Inativo fisicamente & 382 & 70,8 & $66,7-74,4$ \\
\hline Insuficientemente ativo fisicamente & 52 & 9,6 & $7,4-12,4$ \\
\hline Ativo fisicamente & 106 & 19,6 & $16,4-23,2$ \\
\hline \multicolumn{4}{|l|}{ Atividade física (trabalho) } \\
\hline Inativo fisicamente & 81 & 36,3 & $30,2-42,8$ \\
\hline Insuficientemente ativo fisicamente & 18 & 8,1 & $5,1-12,4$ \\
\hline Ativo fisicamente & 124 & 55,6 & $48,9-62,0$ \\
\hline \multicolumn{4}{|l|}{ Tabagismo } \\
\hline Nunca fumou & 276 & 51,1 & $46,8-55,3$ \\
\hline Ex-fumante & 155 & 28,7 & $25,0-32,6$ \\
\hline Fumante & 109 & 20,2 & $17,0-23,7$ \\
\hline \multicolumn{4}{|l|}{ Auto percepção de saúde } \\
\hline Excelente & 26 & 4,8 & $3,2-6,9$ \\
\hline Muito boa & 37 & 6,8 & $4,9-9,3$ \\
\hline Boa & 199 & 36,8 & $32,8-41,0$ \\
\hline Regular & 215 & 39,8 & $35,7-44,0$ \\
\hline Ruim & 63 & 11,8 & $9,2-14,6$ \\
\hline \multicolumn{4}{|l|}{$\begin{array}{l}\text { Diagnóstico médico referido de } \\
\text { hipertensão arterial }\end{array}$} \\
\hline Não & 312 & 57,8 & $53,5-61,8$ \\
\hline $\operatorname{Sim}$ & 228 & 42,2 & $38,1-46,4$ \\
\hline \multicolumn{4}{|l|}{$\begin{array}{l}\text { Diagnóstico médico referido de } \\
\text { diabetes mellitus }\end{array}$} \\
\hline Não & 465 & 86,1 & $82,9-88,7$ \\
\hline Sim & 75 & 13,9 & $11,2-17,0$ \\
\hline \multicolumn{4}{|l|}{$\begin{array}{l}\text { Diagnóstico médico referido de } \\
\text { depressão }\end{array}$} \\
\hline Não & 388 & 72,0 & $68,0-75,6$ \\
\hline Sim & 151 & 28,0 & $24,3-31,9$ \\
\hline \multicolumn{4}{|l|}{ Índice de massa corporal $\left(\mathrm{Kg} / \mathrm{m}^{2}\right)$} \\
\hline Normal & 172 & 33,8 & $29,8-38,0$ \\
\hline Sobrepeso & 186 & 36,2 & $32,1-40,4$ \\
\hline Obesidade & 154 & 30,0 & $26,1-34,0$ \\
\hline \multicolumn{4}{|l|}{ Uso de medicamentos contínuos } \\
\hline Nenhum & 197 & 36,5 & $32,5-40,6$ \\
\hline $1-2$ & 172 & 31,8 & $28,0-35,9$ \\
\hline 3 ou mais & 171 & 31,7 & $27,8-35,7$ \\
\hline
\end{tabular}


Tabela 2 - Descrição das variáveis relacionadas a dor crônica dos usuários das unidades básicas de saúde da zona urbana da cidade de Pelotas, Rio Grande do Sul, 2018 ( $\mathrm{n}=224)$.

\begin{tabular}{|c|c|c|c|}
\hline Variáveis & $\mathrm{n}$ & $\%$ & IC95\% \\
\hline \multicolumn{4}{|l|}{ Interferência da dor } \\
\hline \multicolumn{4}{|l|}{ Período da cronicidade } \\
\hline Dor leve & 17 & 7,6 & $4,7-11,9$ \\
\hline Dor moderada & 66 & 29,5 & $23,8-35,8$ \\
\hline Dor forte & 81 & 36,2 & $30,1-42,7$ \\
\hline Dor insuportável & 41 & 18,2 & $13,7-24,0$ \\
\hline Dor máxima & 19 & 8,5 & $5,4-12,9$ \\
\hline \multicolumn{4}{|l|}{ Momento da entrevista } \\
\hline Sem dor & 66 & 29,5 & $23,8-35,8$ \\
\hline Dor leve & 81 & 36,2 & $30,0-42,7$ \\
\hline Dor moderada & 47 & 21,0 & $16,1-26,8$ \\
\hline Dor forte & 20 & 8,8 & $5,8-13,4$ \\
\hline Dor insuportável & 7 & 3,1 & $1,4-6,4$ \\
\hline Dor máxima & 3 & 1,4 & $0,4-4,1$ \\
\hline \multicolumn{4}{|l|}{ Atividade geral } \\
\hline Não interfere & 46 & 20,5 & $15,7-26,3$ \\
\hline Interfere um pouco & 58 & 25,9 & $20,5-32,0$ \\
\hline Interfere moderadamente & 43 & 19,2 & $14,5-24,9$ \\
\hline Interfere totalmente & 77 & 34,4 & $28,4-40,8$ \\
\hline \multicolumn{4}{|l|}{ Qualidade do sono } \\
\hline Não interfere & 67 & 29,9 & $24,2-36,2$ \\
\hline Interfere um pouco & 47 & 21,0 & $16,1-26,8$ \\
\hline Interfere moderadamente & 37 & 16,5 & $12,1-22,0$ \\
\hline Interfere totalmente & 73 & 32,6 & $26,7-39,0$ \\
\hline \multicolumn{4}{|l|}{ Humor } \\
\hline Não interfere & 75 & 33,5 & $27,5-39,9$ \\
\hline Interfere um pouco & 57 & 25,4 & $20,1-31,6$ \\
\hline Interfere moderadamente & 26 & 11,6 & $8,0-16,5$ \\
\hline Interfere totalmente & 66 & 29,5 & $23,8-35,8$ \\
\hline \multicolumn{4}{|l|}{ Trabalho } \\
\hline Não interfere & 21 & 21,4 & $14,3-30,8$ \\
\hline Interfere um pouco & 30 & 30,6 & $22,1-40,5$ \\
\hline Interfere moderadamente & 12 & 12,3 & $7,0-20,5$ \\
\hline Interfere totalmente & 35 & 35,7 & $26,7-45,8$ \\
\hline \multicolumn{4}{|l|}{ Habilidade para caminhar } \\
\hline Não interfere & 57 & 25,5 & $20,1-31,6$ \\
\hline Interfere um pouco & 55 & 24,5 & $19,3-30,6$ \\
\hline Interfere moderadamente & 36 & 16,1 & $11,7-21,5$ \\
\hline Interfere totalmente & 76 & 33,9 & $27,9-40,4$ \\
\hline \multicolumn{4}{|c|}{ Relacionamento com outras pessoas } \\
\hline Não interfere & 136 & 60,7 & $54,1-66,9$ \\
\hline Interfere um pouco & 28 & 12,5 & $8,7-17,5$ \\
\hline Interfere moderadamente & 15 & 6,7 & $4,0-10,8$ \\
\hline Interfere totalmente & 45 & 20,1 & $15,3-25,8$ \\
\hline \multicolumn{4}{|l|}{ Apreciar a vida } \\
\hline Não interfere & 99 & 44,2 & $37,7-50,8$ \\
\hline Interfere um pouco & 32 & 14,3 & $10,2-19,5$ \\
\hline Interfere moderadamente & 27 & 12,0 & $8,3-17,0$ \\
\hline Interfere totalmente & 66 & 29,5 & $23,8-35,8$ \\
\hline \multicolumn{4}{|l|}{ Tratamentos para alívio da dor } \\
\hline Não & 58 & 25,9 & $20,5-32,0$ \\
\hline $\operatorname{Sim}$ & 166 & 74,1 & $67,9-79,4$ \\
\hline \multicolumn{4}{|c|}{ Tratamento utilizado para dor* } \\
\hline Medicamento & 137 & 82,5 & $75,9-87,6$ \\
\hline Pomada & 28 & 16,9 & $11,8-23,4$ \\
\hline Injeção & 17 & 10,2 & $6,4-15,9$ \\
\hline Fisioterapia & 13 & 7,8 & $4,6-13,1$ \\
\hline Chá & 12 & 7,2 & $4,1-12,4$ \\
\hline
\end{tabular}

Continua...

\begin{tabular}{|c|c|c|c|}
\hline Variáveis & $\mathrm{n}$ & $\%$ & IC95\% \\
\hline \multicolumn{4}{|c|}{ Faltas ao trabalho por causa da dor** } \\
\hline Não & 31 & 31,0 & $22,5-40,8$ \\
\hline Sim & 69 & 69,0 & $59,1-77,4$ \\
\hline \multicolumn{4}{|c|}{ Uso de medicamentos para dor } \\
\hline Não & 34 & 15,2 & $11,0-20,5$ \\
\hline $\operatorname{Sim}$ & 190 & 84,8 & $79,4-88,9$ \\
\hline \multicolumn{4}{|c|}{$\begin{array}{l}\text { Porcentagem de alívio do tratamento ou } \\
\text { medicação para a dor }\end{array}$} \\
\hline $0 \%$ & 9 & 4,4 & $2,3-8,3$ \\
\hline $10 \%$ & 9 & 4,4 & $2,3-8,3$ \\
\hline $20 \%$ & 11 & 5,4 & $3,0-9,5$ \\
\hline $30 \%$ & 6 & 3,0 & $1,3-6,4$ \\
\hline $40 \%$ & 10 & 4,9 & $2,6-8,9$ \\
\hline $50 \%$ & 39 & 19,2 & $14,3-25,2$ \\
\hline $60 \%$ & 17 & 8,4 & $5,2-13,1$ \\
\hline $70 \%$ & 21 & 10,4 & $6,8-15,3$ \\
\hline $80 \%$ & 27 & 13,3 & $9,2-18,7$ \\
\hline $90 \%$ & 18 & 8,9 & $5,6-13,6$ \\
\hline $100 \%$ & 36 & 17,7 & $13,0-23,6$ \\
\hline \multicolumn{4}{|c|}{ Temperatura a qual percebe mais dor } \\
\hline Mais elevadas & 36 & 16,1 & $11,7-21,5$ \\
\hline Mais baixas & 98 & 43,7 & $37,3-50,3$ \\
\hline Não tem relação & 90 & 40,2 & $33,9-46,7$ \\
\hline \multicolumn{4}{|c|}{ Clima o qual percebe mais dor } \\
\hline Seco & 2 & 0,9 & $0,2-3,5$ \\
\hline Úmido & 109 & 48,7 & $42,1-55,2$ \\
\hline Não tem relação & 113 & 50,4 & $43,8-56,9$ \\
\hline
\end{tabular}

*Cinco tratamentos mais prevalentes dos usuários que relataram realizar algum tratamento para dor crônica. $\mathrm{O}$ usuário pode relatar mais de um tratamento. ${ }^{* *}$ Foram considerados apenas os usuários que estavam trabalhando.

nóstico médico referido positivo para hipertensão arterial, diabetes mellitus e depressão e o consumo elevado de medicamentos referidos para o uso contínuo estiveram associados com a dor crônica. Ser ativo no lazer foi fator de proteção para o evento dor crônica. $\mathrm{Na}$ análise ajustada, permaneceram associadas ao desfecho o sexo feminino (50\% maior probabilidade em relação aos homens), o aumento da idade (usuários com 40-59 anos uma maior probabilidade de $44 \%$ e os de $60-90$ anos maior probabilidade de $61 \%$ do que os de $18-39$ anos), a auto percepção de saúde negativa (aqueles que relataram a sua saúde como ruim, tiveram 5,4 vezes mais probabilidade de dor crônica comparados com os usuários que relataram sua saúde com excelente ou muito boa), o uso elevado de medicamentos referidos para o uso contínuo (usuários que utilizavam três ou mais medicamentos, tiveram maior probabilidade de $43 \%$ em relação aos que não utilizavam medicamentos) e a atividade física de lazer (os usuários insuficientemente ativos tiveram uma menor probabilidade de $24 \%$ e os ativos uma menor probabilidade de $22 \%$ em relação aos indivíduos inativos) - Tabela 3 . 
Tabela 3 - Prevalência, análise bruta e ajustada do desfecho dor crônica em relação às variáveis independentes, para usuários das unidades básicas de saúde da zona urbana da cidade de Pelotas, Rio Grande do Sul, 2018 ( $\mathrm{n}=540$ ).

\begin{tabular}{|c|c|c|c|c|c|}
\hline \multirow{2}{*}{ Variáveis } & \multirow{2}{*}{$\%$} & Análise bruta & \multirow[b]{2}{*}{$\mathrm{p}$} & Análise ajustada & \multirow[b]{2}{*}{$\mathrm{p}$} \\
\hline & & $\mathrm{RP}(\mathrm{IC} 95 \%)^{* * * *}$ & & $\mathrm{RP}(\mathrm{IC} 95 \%)^{* * * *}$ & \\
\hline Sexo & & & 0,002 & & 0,003 \\
\hline Masculino & 30,6 & 1,00 & & 1,00 & \\
\hline Feminino & 45,5 & $1,49(1,14-1,94)$ & & $1,50(1,15-1,97)$ & \\
\hline Faixa etária & & & $0,001^{*}$ & & $<0,001$ \\
\hline $18-39$ & 31,3 & 1,00 & & 1,00 & \\
\hline $40-59$ & 44,6 & $1,43(1,09-1,85)$ & & $1,44(1,11-1,88)$ & \\
\hline $60-90$ & 49,7 & $1,59(1,20-2,08)$ & & $1,61(1,23-2,10)$ & \\
\hline Cor da pele & & & 0,256 & & 0,522 \\
\hline Branca & 43,0 & 1,00 & & 1,00 & \\
\hline Não branca & 37,6 & $0,87(0,69-1,10)$ & & $0,93(0,73-1,17)$ & \\
\hline Situação Conjugal & & & 0,861 & & 0,488 \\
\hline Sem companheiro (a) & 42,0 & 1,00 & & 1,00 & \\
\hline Com companheiro (a) & 41,3 & $0,98(0,80-1,20)$ & & $1,07(0,87-1,32)$ & \\
\hline Renda mensal (salários mínimos) & & & $0,920^{*}$ & & $0,604^{*}$ \\
\hline Menos de 1 & 39,0 & 1,00 & & 1,00 & \\
\hline De 1 até 2 & 45,6 & $1,17(0,94-1,45)$ & & $1,08(0,87-1,35)$ & \\
\hline 2 ou mais & 31,7 & $0,81(0,54-1,22)$ & & $0,79(0,52-1,20)$ & \\
\hline Tabagismo & & & $0,211^{*}$ & & 0,093* \\
\hline Nunca fumou & 39,1 & 1,00 & & 1,00 & \\
\hline Ex-fumante & 42,6 & $1,09(0,86-1,38)$ & & $1,07(0,85-1,35)$ & \\
\hline Fumante & 45,9 & $1,17(0,91-1,50)$ & & $1,25(0,98-1,60)$ & \\
\hline Situação profissional & & & 0,115 & & 0,618 \\
\hline Aposentado & 50,0 & 1,00 & & 1,00 & \\
\hline Não trabalha & 40,3 & $0,80(0,63-1,03)$ & & $0,85(0,65-1,12)$ & \\
\hline Trabalha & 38,2 & $0,76(0,59-0,99)$ & & $0,90(0,68-1,19)$ & \\
\hline Índice de massa corporal & & & $0,294^{*}$ & & $0,685^{*}$ \\
\hline Normal & 39,7 & 1,00 & & 1,00 & \\
\hline Sobrepeso & 40,9 & $1,03(0,80-1,32)$ & & $1,06(0,84-1,34)$ & \\
\hline Obesidade & 45,5 & $1,14(0,89-1,47)$ & & $1,50(0,83-1,33)$ & \\
\hline Auto percepção de saúde & & & $<0,001^{*}$ & & $<0,001$ \\
\hline Excelente/Muito boa & 12,7 & 1,00 & & 1,00 & \\
\hline Boa & 24,6 & $1,93(0,97-3,87)$ & & $1,87(0,94-3,72)$ & \\
\hline Regular & 54,9 & $4,32(2,23-8,35)$ & & $3,98(2,05-7,74)$ & \\
\hline Ruim & 77,8 & $6,12(3,16-11,87)$ & & $5,41(2,76-10,60)$ & \\
\hline Hipertensão arterial & & & 0,028 & & 0,598 \\
\hline Não & 37,4 & 1,00 & & 1,00 & \\
\hline Sim & 46,9 & $1,25(1,02-1,53)$ & & $0,94(0,76-1,17)$ & \\
\hline Diabetes mellitus & & & 0,025 & & 0,859 \\
\hline Não & 39,6 & 1,00 & & 1,00 & \\
\hline Sim & 53,3 & $1,35(1,06-1,71)$ & & $1,02(0,81-1,29)$ & \\
\hline Depressão & & & $<0,001$ & & 0,673 \\
\hline Não & 35,6 & 1,00 & & 1,00 & \\
\hline Sim & 56,3 & $1,58(1,30-1,92)$ & & $1,04(0,85-1,28)$ & \\
\hline Uso de medicamentos & & & $<0,001^{*}$ & & $0,039^{*}$ \\
\hline Nenhum & 24,9 & 1,00 & & 1,00 & \\
\hline Entre 1 ou 2 & 46,5 & $1,87(1,40-2,50)$ & & $1,47(1,10-1,97)$ & \\
\hline 3 ou mais & 55,6 & $2,23(1,69-2,95)$ & & $1,43(1,04-1,95)$ & \\
\hline
\end{tabular}

Continua... 


\begin{tabular}{|c|c|c|c|c|c|}
\hline \multirow{2}{*}{ Variáveis } & \multirow{2}{*}{$\%$} & Análise bruta & \multirow{2}{*}{$\mathrm{p}$} & Análise ajustada & \multirow{2}{*}{$\mathrm{p}$} \\
\hline & & $\mathrm{RP}(\mathrm{IC} 95 \%)^{* * *}$ & & RP (IC95\%)**** & \\
\hline${\text { Atividade física (lazer) }{ }^{* *}}^{*}$ & & & $0,004^{*}$ & & $0,047^{*}$ \\
\hline Inativo fisicamente & 45,3 & 1,00 & & 1,00 & \\
\hline Insuficientemente ativo fisicamente & 36,5 & $0,80(0,55-1,17)$ & & $0,76(0,53-1,07)$ & \\
\hline Ativo fisicamente & 30,2 & $0,66(0,49-0,90)$ & & $0,78(0,58-1,04)$ & \\
\hline Atividade física (trabalho) ${ }^{* *}$ & & & $0,597^{*}$ & & $0,301^{*}$ \\
\hline Inativo fisicamente & 37,0 & 1,00 & & 1,00 & \\
\hline Insuficientemente ativo fisicamente & 27,8 & $0,75(0,34-1,67)$ & & $1,0(0,48-2,11)$ & \\
\hline Ativo fisicamente & 40,3 & $1,09(0,76-1,55)$ & & $1,20(0,84-1,70)$ & \\
\hline
\end{tabular}

Modelo hierárquico: $1^{\circ}$ nível - sexo, idade, cor da pele, situação conjugal e renda mensal; $2^{\circ}$ nível - tabagismo; $3^{\circ}$ nível - situação profissional; $4^{\circ}$ nível - Índice de massa corporal e auto percepção de saúde; $5^{\circ}$ nível - diagnóstico médico de hipertensão, diabetes e depressão; $6^{\circ}$ nível número de medicamentos de uso contínuo; $7^{\circ}$ nível - atividade física de lazer/atividade física de trabalho. Após a retirada das variáveis com $\mathrm{p}$ $\leq 0,20$, o modelo final ficou com as seguintes variáveis: sexo, idade, fumo, auto percepção de saúde, número de medicamentos e atividade física de lazer e trabalho. *Valores de p calculados pelo teste de Wald de tendência linear. **A atividade física de lazer e de trabalho foram adicionadas ao modelo separadamente, devido ao efeito particular de cada uma. $\quad{ }^{* * *} \mathrm{RP}=$ razão de prevalência; $\mathrm{IC} 95 \%=$ intervalo de confiança $95 \%$.

\section{Discussão}

A prevalência de dor crônica encontrada nesse estudo foi de $41,5 \%$, e a atividade física de lazer foi fator de proteção para o surgimento de dor crônica. Ainda são escassos os estudos relacionados à dor crônica nas UBS. Em Santa Maria, Rio Grande do Sul, um estudo realizado em uma UBS, constatou uma prevalência de dor crônica de $37,8 \%{ }^{17}$. Resultados semelhantes ao presente estudo, foram encontrados em estudos populacionais, como na cidade de Salvador, Bahia $(41,4 \%)^{18}$ e São Luís, Maranhão $(42 \%)^{19}$. Esses dados estão de acordo com os achados a nível mundial em países de baixa e média renda e com a prevalência média relatada pela $\operatorname{IASP}(35,5 \%)^{13}$. A dor crônica gera um forte impacto na vida das pessoas, reflete de maneira negativa nas tarefas diárias, tanto físicas quanto sociais, sendo que muitas pessoas são diagnosticadas com depressão por causa da dor ${ }^{7}$.

A intensidade da dor é importante para avaliar a dor crônica na sua complexidade. O presente estudo revelou que $92,4 \%$ dos usuários entrevistados classificaram a sua dor como moderada, forte, insuportável ou máxima, durante o período igual ou superior a três meses de dor. Uma prevalência elevada na intensidade da dor crônica também foi encontrada em um estudo realizado no Canadá ${ }^{20}$ e em outro nos Estados Uni$\operatorname{dos}^{21}$, em ambos, $80 \%$ dos relatos foram referentes à intensidade moderada ou severa. Moulin et al. ${ }^{20}$, mencionaram que pacientes com dor crônica costumavam tomar analgésicos prescritos e os que possuíam intensidades de dor mais elevadas, provavelmente eram direcionados para terapias com opióides por não obterem eficácia no tratamento convencional. Nestes estudos, o período considerado para a dor crônica foi de seis me- ses. Cabe mencionar a influência de um possível viés recordatório em relação aos resultados desses estudos, devido aos distintos períodos considerados para a cronicidade da dor.

Enquanto no período de cronicidade de três meses ou mais a intensidade da dor foi classificada na maioria dos casos como forte, insuportável ou máxima $(62,9 \%)$, no momento da entrevista, ela foi considerada como leve ou sem dor (65,7\%). Prevalências semelhantes para sensações de intensidade extremamente distintas poderiam estar relacionadas ao elevado uso de medicamentos para a dor por parte dos usuários $(84,8 \%)$ que, quando faziam efeito, aliviavam fortemente os sintomas. Elevado consumo de analgésicos também foi encontrado em outros estudos ${ }^{7,19}$. Apesar do elevado uso de medicamentos para tratamento da dor apontado neste estudo, $19,2 \%$ dos entrevistados relataram sentir apenas 50,0\% de alívio e, secundariamente, $17,7 \%$ relataram $100 \%$ de alívio. Seu uso indiscriminado, pode apresentar efeitos adversos, que variam desde os mais leves como boca seca, sonolência, prisão de ventre, tontura, alergia, dor de estômago ${ }^{22}$, até a morte causada por uma úlcera perfurada ou hemorragia ${ }^{7}$. Desde 2006, as chamadas Práticas Integrativas e Complementares em Saúde encontram-se em processo de implantação crescente nas UBS de todo o país, como uma forma de terapia não medicamentosa e reconhecida internacionalmente pela Organização Mundial da Saúde e por diversos países ${ }^{23}$.

A prática de atividade física também faz parte da atenção básica ${ }^{11}$ e possui importante contribuição no tratamento das dores crônicas. Ela é essencial para o equilíbrio neuro-hormonal e ativação dos mecanismos endógenos de controle da dor em pessoas sadias ${ }^{24}$, po- 
dendo ser de intensidade moderada, no mínimo durante dez minutos e em uma frequência trissemanal ${ }^{14}$.

Pesquisas identificaram algumas das interferências da dor crônica relatadas neste estudo, tais como, na qualidade do sono $^{25}$, no trabalho ${ }^{19}$, na habilidade para caminhar ${ }^{25}$, também no relacionamento com outras pessoas $^{20}$, para apreciar a vida ${ }^{20}$ e no humor ${ }^{19}$. Contudo, a dor crônica causa um grande impacto social e econômico, sendo necessário um programa abrangente de tratamento que inclua abordagens em terapias físicas, psicológicas e comportamentais para o controle da dor ${ }^{20}$.

Um estudo ${ }^{7}$ mostrou que $61,0 \%$ das pessoas encontraram-se pouco capacitadas ou incapacitadas para trabalhar fora de casa, 19,0\% tinham perdido seu trabalho e 13,0\% tinham mudado seu trabalho por causa da dor. $\mathrm{O}$ presente estudo também apontou prevalência elevada de usuários que relataram ter faltado ao trabalho por causa da dor, porém, somente em relação às faltas ocasionadas pela dor naqueles que estavam trabalhando fora de casa. As pessoas que estavam afastadas do trabalho por motivo de dor/doença ou que recebiam auxílio doença, não foram consideradas as faltas, o que sugere que a prevalência de faltas ao trabalho por dor crônica poderia ser maior.

As regiões álgicas mais afetadas pela dor crônica encontradas neste estudo, lombar $(28,6 \%)$, joelhos $(21,0 \%)$, cabeça $(14,3 \%)$, cervical $(13,4 \%)$, ombros $(8,9 \%)$ e torácica $(8,9 \%)$, são semelhantes aos locais mais afetados pela dor crônica encontrados por Moulin et al. ${ }^{20}$, sendo que em vários estudos a região lombar foi a mais citada ${ }^{3,18,21}$, seguida dos membros e da região da cabeça $\mathrm{a}^{19}$. Um estudo de revisão sistemática e meta-análise global ${ }^{3}$, mencionou que a presença de dor lombar em trabalhadores também é a mais relatada e deve levar em consideração uma abordagem mais difundida com a síndrome de dor crônica e sua associação psicológica, considerando que o tratamento neste caso seria mais complexo do que para lombalgia aguda. Outro estudo ${ }^{19}$, alertou quanto a importância da dor crônica nos membros, tanto inferiores quanto superiores, sendo que estes locais podem estar possivelmente associados a doenças tais como o diabetes, compressão radicular, entre outras.

Poucas pesquisas envolvem as variáveis temperatura e clima, sendo estas um motivo de queixa das pessoas com dor crônica ${ }^{16}$. Assim, 43,7\% dos usuários perceberam o aumento das suas dores em temperaturas mais baixas e $48,7 \%$ em clima úmido, o que sugere uma relação entre temperaturas mais baixas e umidade, gerando assim uma maior contração muscular, compressão ar- ticular e tensão, ocasionando em dores ${ }^{26}$. Porém, ainda são necessários mais estudos na obtenção de evidências relacionadas as atividades diárias e qualidade de vida ${ }^{16}$.

$\mathrm{Na}$ análise ajustada, ser do sexo feminino esteve associada ao desfecho dor crônica, o que ficou a concorde com vários estudos ${ }^{5,19-21}$. Possivelmente a mulher sofra com mais dor devido a alterações hormonais, nutricionais e fatores psicológicos ${ }^{27}$. $\mathrm{O}$ aumento da idade também ficou associado na análise ajustada ${ }^{21,25}$, sendo este um processo natural de envelhecimento no qual, ao longo da vida, ocorrem mudanças nas funções fisiológicas, assim como também o aumento do risco de doenças ${ }^{25}$. A permanência da associação da auto percepção de saúde negativa na análise ajustada ${ }^{28}$ retrata que as pessoas que consideram sua condição de saúde regular ou ruim, é porque supostamente já possuem alguma doença e, consequentemente, sofra de dor por este motivo ${ }^{29}$. Outra variável que esteve associada a dor crônica na análise ajustada foi o uso elevado de medicamentos referidos para uso contínuo, sendo este um viés de causalidade reversa do estudo. Não foram encontrados na literatura estudos relacionando especificamente a esta variável com o desfecho. No entanto, sabe-se que para o tratamento e alívio da dor, os medicamentos são frequentemente utilizados, o que pode explicar tal associação.

Foi verificada uma tendência em relação à prática de atividade física no lazer e dor crônica, sendo que conforme modificava a categoria de inativo para insuficientemente ativo e ativo, diminuía a probabilidade do usuário manifestar dor crônica. No entanto, levando em consideração o delineamento transversal do estudo, é possível que haja causalidade reversa no resultado, sendo que pessoas que sentem menos dor podem fazer mais atividade física ou então quem faz mais atividade física sente menos dor. Um estudo em larga escala realizado em quarenta e seis países de baixa e média ren$\mathrm{da}^{30}$ revelou que muitas condições crônicas estiveram associadas com a baixa prática de atividade física. Nesse sentido, torna-se de suma importância que os usuários de UBS recebam orientação e suporte para a prática de atividade física, tendo em vista que as UBS devem desenvolver ações de prevenção e tratamento de doenças e promoção da saúde. Considerando que a grande maioria dos usuários são de baixa renda e muitas vezes a UBS se configura como o principal local para o recebimento de ações de educação em saúde, estratégias para estas ações devem ser implementadas.

Algumas limitações deste estudo devem ser consideradas. $\mathrm{O}$ delineamento transversal, não permite de- 
finir relações de causalidade entre a prevalência de dor crônica e algumas variáveis investigadas, assim como as medidas auto referidas das variáveis estudadas podem causar viés de memória. Dentre os pontos fortes do estudo está o fato das UBS estarem bem distribuídas em áreas estratégicas na cidade, garantindo equanimidade na amostra e representatividade. Levando em consideração as características similares de usuários de UBS de diferentes regiões e os métodos empregados no presente estudo, os resultados obtidos devem ser considerados relevantes para outras localidades.

Conclui-se que dentre os usuários das UBS do município de Pelotas, Rio Grande do Sul, existe uma alta prevalência de dor crônica, sendo ela maior entre as mulheres, aumentando de acordo com a idade, com o número elevado de medicamentos de uso contínuo, com a percepção de saúde negativa e com a inatividade física de lazer. Torna-se importante o papel do profissional de educação física e das práticas de atividade física nas UBS como forma de promoção da saúde e prevenção de doenças, considerando a dor crônica um problema recorrente dos usuários. Além disso, existe a importância da capacitação profissional adequada no acolhimento ao usuário com dor crônica, de estudos de intervenção e equipe multiprofissional.

\section{Conflito de interesses}

Os autores declaram não haver conflito de interesses.

\section{Contribuição dos autores}

Souza DFS, participou da elaboração do projeto e de todas as demais etapas até a redação final do artigo, incluindo a coleta e análise de dados. Häfele $V$, participou da coleta e análise de dados, além da revisão final do artigo. Siqueira FV, participou na qualidade de revisor final de todas as etapas desde a elaboração do projeto até a redação final do artigo.

\section{Agradecimentos}

Os autores agradecem a Escola Superior de Educação Física da Universidade Federal de Pelotas, a Secretaria Municipal de Saúde de Pelotas, as Unidades Básicas de Saúde e a todos os envolvidos que tornaram possível a realização deste estudo. A Fundação de Amparo à Pesquisa do Estado do Rio Grande do Sul (FAPERGS), pela bolsa de doutorado do autor VH.

\section{Referências}

1. Ministério da Saúde. Política Nacional de Atenção Básica [Internet]. Série E Legislação em Saúde. 2012. 114 p. Disponível em: http://189.28.128.100/dab/docs/publicacoes/ geral/pnab.pdf.
2. Assadeck H, Daouda MT, Djibo FH, Maiga DD, Omar EA. Prevalence and characteristics of chronic pain: Experience of Niger. Scand J Pain. 2017;17(1):252-55.

3. Jackson T, Thomas S, Stabile V, Shotwell M, Han X, McQueen K. A systematic review and meta-analysis of the global burden of chronic pain without clear etiology in lowand middle-income countries: trends in heterogeneous data and a proposal for new assessment methods. Anesth Analg. 2016;123(3):739-48.

4. Cipriano A, Almeida DB, Vall J. Perfil do paciente com dor crônica atendido em um ambulatório de dor de uma grande cidade do sul do Brasil. Rev Dor. 2011;12 (4):297- 300.

5. Teixeira MJ. Master dor. $1^{a}$ ed. São Paulo: Limay, 1994.

6. Breivik H, Collett B, Ventafridda V, Cohen R, Gallacher D. Survey of chronic pain in Europe: Prevalence, impact on daily life, and treatment. Eur J Pain. 2006;10:287-33.

7. Sociedade Brasileira para Estudo da Dor. Brasil sem dor [Internet]. Campanha nacional pelo tratamento e controle da dor aguda e crônica. 2018. 11 p. Disponível em: https:// sbed.org.br/wp-content/uploads/2019/01/CAMPANHANACIONAL-PELO-TRATAMENTO-E-CONTROLEDA-DOR-AGUDA-E-CR\%C3\%94NICA-3-MB.pdf.

8. Holth HS, Werpen HKB, Zwart J-A, Hagen K. Physical inactivity is associated with chronic musculoskeletal complaints 11 years later: results from the Nord-Trøndelag Health Study. BMC Musculoskelet Disord. 2008;9:159.

9. Paley CA, Johnson MI. Physical activity to reduce systemic inflammation associated with chronic pain and obesity a narrative review. Clin J Pain. 2016;32(4):365-70.

10. Ministério da Saúde. Diretrizes do NASF: Núcleo de Apoio à Saúde da Família [Internet]. Cadernos de Atenção Básica. 2010. 152 p. Disponível em: http://189.28.128.100/dab/ docs/publicacoes/cadernos_ab/abcad27.pdf.

11. Souza SC, Loch MR. Intervenção do profissional de educação física nos Núcleos de Apoio à Saúde da Família em municípios do norte do Paraná. Rev Bras Ativ Fís Saúde. 2011;16(1):5-10.

12. Vieira A, Bartz PT, Jornada MC. Repercussão do grupo da coluna sobre o cotidiano de mulheres que apresentam dores. Cad Bras Ter Ocup. 2017;25(2):305-14.

13. World Health Organization. Global recommendations on physical activity for health [Internet]. 2010.60 p. Disponível em: https://www.who.int/dietphysicalactivity/global-PArecs-2010.pdf.

14. Harstall C, Ospina M. How prevalent is chronic pain? Pain. 2003;11(2):1-4.

15. Wong D, Baker C. Pain in Children: Comparison of Assessment Scales. Pediatr Nursing. 1988;14(1):9-17.

16. Figueiredo ECQ, Figueiredo GC, Dantas RT. Influência de elementos meteorológicos na dor de pacientes com osteoartrite: revisão da literatura. Rev Bras Reumatol. 2011;51(6):616-28.

17. Ruviaro LF, Filippin LI. Prevalência de dor crônica em uma Unidade Básica de Saúde de cidade de médio porte. Rev Dor. 2012;13(2):128-31.

18. Sá KN, Baptista AF, Matos MA, Lessa Í. Chronic pain and gender in Salvador population, Brazil. Pain. 2008;139:498-506.

19. Vieira ÉBM, Garcia JBS, Silva AAM, Araújo RLTM, Jansen RCS. Prevalence, characteristics, and factors associated with chronic pain with and without neuropathic characteristics in São Luís, Brazil.J Pain Symptom Manage. 2012;44(2):239-51.

20. Moulin DE, Clark AJ, Speechley M, Morley-Forster PK. Chronic pain in Canada - Prevalence, treatment, impact and the role of opioid analgesia. Pain Res Manag. 2002;7(4):179-84. 
21. Johannes CB, Le TK, Zhou X, Johnston JA, Dworkin RH. The Prevalence of chronic pain in United States adults: results of an internet-based survey. J Pain. 2010;11(11):1230-9.

22. Hospital Erasto Gaertner. Serviço de Tratamento da Dor. Setor de Farmácia Cartilha de Orientação dos Medicamentos para Dor [Internet].2008. 14 p. Disponível em: http://www.dol.inf.br/Html/CartilhaErastoGaertner/ CartilhaErastoGaertner.pdf.

23. Ministério da Saúde. Glossário temático: Práticas integrativas e complementares em saúde [Internet]. 2018. 181 p. Disponível em: https://portalarquivos2.saude.gov.br/images/ pdf/2018/marco/12/glossario-tematico.pdf.

24. Souza JB. Poderia a atividade física induzir analgesia em pacientes com dor crônica? Rev Bras Med Esp. 2009;15(2):145-50.

25. Jakobsson U. The epidemiology of chronic pain in a general population: Results of a survey in southern Sweden. Scand J Rheumatol. 2010;39:421-9.

26. Tanaka C. Pacientes portadores de fibromialgia: efeito sobre mobilidade, dor e sono. Rev Fisio. 1994;1(1):16-20.
27. Manson JE. Pain: Sex differences and implications for treatment. Metab Clinand Exprim. 2010;59(1):16-20.

28. Sjøgren P, Ekholm O, Peuckmann V, Grønbæk M. Epidemiology of chronic pain in Denmark: An update. Eur J Pain. 2009;13:287-92.

29. Medeiros SM, Silva LSR, Carneiro JA, Ramos GCF, Barbosa ATF, Caldeira AP. Fatores associados à autopercepção negativa da saúde entre idosos não institucionalizados de Montes Claros, Brasil. Cien Saude Colet. 2016;21(11):3377-86.

30. Vancampfort D, Koyanagi A, Ward PB, Rosenbaum S, Schuch FB, Mugisha J, et al. Chronic physical conditions, multimorbidity and physical activity across 46 low- and middle-income countries. Int J Behav Nutr Phys Act. 2017;14(6):1-13.

Recebido: 26/05/2019

Aprovado: 14/11/2019

\section{este artigo:}

Souza DFS, Häfele V, Siqueira FV. Dor crônica, nivel de atividade física e fatores associados em usuários das unidades básicas de saúde. Rev Bras Ati Fis Saúde. 2019;24:e0085. DOI: 10.12820/rbafs.24e0085 